\title{
A VARIATIONAL APPROACH OF THE STURM-LIOUVILLE PROBLEM IN FRACTIONAL DIFFERENCE CALCULUS
}

\author{
RAZIYE MERT ${ }^{1}$, LYNN ERBE $^{2}$, AND THABET ABDELJAWAD ${ }^{3}$ \\ ${ }^{1}$ Mechatronic Engineering Department \\ University of Turkish Aeronautical Association \\ 06790, Ankara, TURKEY \\ ${ }^{2}$ Department of Mathematics \\ University of Nebraska-Lincoln \\ Lincoln, NE, 68588-0130, USA \\ ${ }^{3}$ Department of Mathematics and General Sciences \\ Prince Sultan University \\ P.O. Box 66833, 11586 Riyadh, SAUDI ARABIA
}

\begin{abstract}
In this article, we formulate and analyze a nabla fractional difference Sturm Liouville problem $(S L P)$ with the nabla left Caputo fractional difference and the nabla right Riemann-Liouville fractional difference. The discrete fractional variational calculus is used to study the eigenvalues and eigenfunctions of the formulated $S L P$ by presenting a new nabla fractional difference isoperimetric variational problem.
\end{abstract}

AMS Subject Classification: 35R11, 34A08

Key Words: nabla left Caputo fractional difference, nabla right Riemann-Liouville fractional difference, integration by parts, discrete fractional isoperimetric problem, Sturm-Liouville problem

Received: 2017-11-05;

Published: January 26, 2018
Accepted: 2018-01-16 ;

doi: $\quad 10.12732 /$ dsa.v27i1.7

https://acadsol.eu/dsa

\section{INTRODUCTION AND PRELIMINARIES}

The theory of fractional calculus and its role in the development of dynamical systems of arbitrary order has been under investigation for a long time. Such fractional 
dynamical systems have been used effectively in the modelling of many real world problems in various fields of science and engineering, where they succeeded to reflect the description of the properties of non-local complex systems $[1,2,3,4,5]$. On the other hand, the discrete fractional calculus has shown much interest among researchers in the last two decades $[6,7,8,9,10,11,12,13]$ and has been developing rapidly, where the integration by parts problem has been formulated via left and right fractional differences and sums.

The calculus of variation which deals mainly with the optimization problems on infinite dimensional function spaces is an extremely important field in mathematics and physics where many geometrical and physical problems can be analyzed by means of variational problems [14]. The issue of obtaining integration by parts formulas in fractional calculus or discrete fractional calculus is the key to obtain fractional Euler-Lagrange equations. In the last decade many researchers studied fractional and discrete fractional problems by applying suitable integration by parts formulas $[15,16,17,18,19,20,21,22,23,24,25,26,27,28,29,30,31]$.

The ordinary $S L E s$ were investigated long ago where many applications in various areas of science, engineering, and mathematics have been reported [32, 33]. However, the fractional variational SLEs have only recently been investigated for local and nonlocal fractional operators $[34,35,36]$.

In this article we use a nabla fractional difference integration by parts formula presented in [15] to formulate a nabla discrete fractional $S L E$ through left and right Caputo and Riemann fractional differences. Then, we continue to study the new formulated $S L P$ by making use of a discrete fractional isoperimetric variational problem. Below, we present some basic tools to be used and then in the following section we proceed to our main results.

For $a, b \in \mathbb{R}$, the sets $\mathbb{N}_{a},{ }_{b} \mathbb{N}, \mathbb{N}_{a, b}$, where $b-a$ is a positive integer, are defined by

$$
\mathbb{N}_{a}=\{a, a+1, a+2, \ldots\}, \quad{ }_{b} \mathbb{N}=\{\ldots, b-2, b-1, b\}
$$

and

$$
\mathbb{N}_{a, b}=\{a, a+1, a+2, \ldots, b\} .
$$

The following integration by parts formula will be essential for proving the main results. It will be used to develop a discrete fractional isoperimetric variational problem to study nabla discrete fractional SLEs.

Theorem 1. [15] Let $0<\alpha \leq 1$ and $f, g: \mathbb{N}_{a, b} \rightarrow \mathbb{R}, a, b \in \mathbb{R}$. Then

$$
\sum_{t=a+1}^{b-1} g(t)\left({ }_{a}^{C} \nabla^{\alpha} f\right)(t)=\left.f(t)\left({ }_{b} \nabla^{-(1-\alpha)} g\right)(t)\right|_{a} ^{b-1}+\sum_{t=a+1}^{b-1} f(t-1)\left({ }_{b} \nabla^{\alpha} g\right)(t-1),
$$

where clearly $\left({ }_{b} \nabla^{-(1-\alpha)} g\right)(b-1)=g(b-1)$. 
In Theorem 1 above, ${ }_{a}^{C} \nabla^{\alpha}$ and $\nabla_{b}^{\alpha}$ represent the left Caputo fractional difference starting from $a$ and the right Riemann-Liouville fractional difference ending at $b$.

\section{MAIN RESULTS}

Our main results consist of two parts. First, we formulate a nabla fractional difference $S L P$ by making use of Theorem 1 . Then, we construct a discrete fractional isoperimetric variational problem to study the eigenvalue and eigenfunctions of the formulated $S L P$.

\subsection{THE DISCRETE FRACTIONAL $S L P$}

In what follows, $\alpha \in \mathbb{R}$ and $0<\alpha \leq 1$ and $b=a+N+1$ for some $N \geq 2$ a fixed integer. Moreover, we set

$$
a_{i}^{(\alpha)}= \begin{cases}1, & \text { if } i=0 \\ (-1)^{i} \frac{\alpha(\alpha-1) \ldots(\alpha-i+1)}{i !}, & \text { if } i=1,2, \ldots\end{cases}
$$

Denoting the Sturm-Liouville operator as

$$
L x(t)=\left({ }_{b} \nabla^{\alpha}\left(p{ }_{a}^{C} \nabla^{\alpha} x\right)\right)(t)+q(t) x(t),
$$

consider the fractional $S L E$

$$
L x(t)=\lambda r(t) x(t), \quad t \in \mathbb{N}_{a+1, b-2},
$$

where $0<\alpha \leq 1, p: \mathbb{N}_{a, b-1} \rightarrow \mathbb{R}, r: \mathbb{N}_{a+1, b-2} \rightarrow \mathbb{R}$ such that $p(t)>0, r(t)>0$ and $q: \mathbb{N}_{a+1, b-2} \rightarrow \mathbb{R}$ with the boundary conditions

$$
x(a)=0, \quad x(b-1)=0 .
$$

Theorem 2. The SLP (2)-(3) has $N-1$ real eigenvalues, which will be denoted by

$$
\lambda_{1} \leq \lambda_{2} \leq \cdots \leq \lambda_{N-1}
$$

The corresponding eigenfunctions,

$$
x_{1}, x_{2}, \ldots, x_{N-1}: \mathbb{N}_{a+1, b-2} \rightarrow \mathbb{R},
$$

are mutually orthogonal with respect to the weight function $r$ on $\mathbb{N}_{a+1, b-2}$; that is,

$$
<x_{i}, x_{j}>_{r}=\sum_{t=a+1}^{b-2} r(t) x_{i}(t) x_{j}(t)=0, \quad i \neq j,
$$


and they span $\mathbb{R}^{N-1}$; that is, any function $\psi: \mathbb{N}_{a+1, b-2} \rightarrow \mathbb{R}$ has the unique representation

$$
\psi(t)=\sum_{i=1}^{N-1} c_{i} x_{i}(t), \quad t \in \mathbb{N}_{a+1, b-2},
$$

where the coefficient $c_{i}$ 's are given by

$$
c_{i}=\frac{<\psi, x_{i}>_{r}}{<x_{i}, x_{i}>_{r}}, \quad 1 \leq i \leq N-1 .
$$

Proof. Note that equations (2) and (3) can be considered as a system of $N-1$ linear equations in $N-1$ real unknowns $x(a+1), x(a+2), \ldots, x(b-2)$. It can be shown that the corresponding matrix form is

$$
A x^{T}=\lambda R x^{T}
$$

where the entries $A_{i j}$ 's of $A$ are given by

$$
A_{i j}^{(\alpha)}=\left\{\begin{array}{l}
q(a+i)+\sum_{k=0}^{N-i}\left(a_{k}^{(\alpha)}\right)^{2} p(a+i+k), \quad i=j \\
\sum_{k=0}^{N-i}\left[a_{k}^{(\alpha)} p(a+i+k) \sum_{m=0}^{k+i} a_{m}^{(\alpha)}\right] \text { and } k-m+i=j, i \neq j,
\end{array}\right.
$$

and $R=\operatorname{diag}(r(a+1), r(a+2), \ldots, r(b-2))$. Because of the equivalence of the $S L P$ (2)-(3) with the problem (4), it follows from the matrix theory that the $S L P(2)$ (3) has $N-1$ pairwise orthogonal real linearly independent eigenfunctions with all eigenvalues real. Now, we will find the constants $c_{i}$ 's. We have

$$
<\psi, x_{j}>_{r}=<\sum_{i=1}^{N-1} c_{i} x_{i}, x_{j}>_{r}=\sum_{i=1}^{N-1} c_{i}<x_{i}, x_{j}>_{r}=c_{j}<x_{j}, x_{j}>_{r},
$$

which follows from the orthogonality. Hence, $c_{i}=\frac{\left\langle\psi, x_{i}\right\rangle_{r}}{\left\langle x_{i}, x_{i}\right\rangle_{r}}, 1 \leq i \leq N-1$, as claimed.

\subsection{THE DISCRETE FRACTIONAL ISOPERIMETRIC PROBLEM}

Let $J$ be a functional of the form

$$
J(f)=\sum_{t=a+1}^{b-1} L\left(t, f^{\rho}(t),\left({ }^{C} \nabla_{a}^{\alpha} f\right)(t)\right),
$$

where $0<\alpha \leq 1, f: \mathbb{N}_{a, b-1} \rightarrow \mathbb{R}, L: \mathbb{N}_{a+1, b-1} \times \mathbb{R} \times \mathbb{R} \rightarrow \mathbb{R}$ such that $L$ has continuous first order partial derivatives with respect to $f^{\rho}$ and ${ }^{C} \nabla_{a}^{\alpha} f$. The isoperimetric problem consists of finding the local extrema of $J$ subject to the boundary conditions

$$
f(a)=A, \quad f(b-1)=B,
$$


where $A, B$ are constants and the isoperimetric constraint

$$
I(f)=\sum_{t=a+1}^{b-1} g\left(t, f^{\rho}(t),\left({ }^{C} \nabla_{a}^{\alpha} f\right)(t)\right)=l,
$$

where $l$ is a constant and $g: \mathbb{N}_{a+1, b-1} \times \mathbb{R} \times \mathbb{R} \rightarrow \mathbb{R}$ such that $g$ has continuous first order partial derivatives with respect to $f^{\rho}$ and ${ }^{C} \nabla_{a}^{\alpha} f$.

Definition 1. A function $f: \mathbb{N}_{a, b-1} \rightarrow \mathbb{R}$ which satisfies (6) and (7) is called admissible.

Definition 2. An admissible function $f$ is called an extremal for $I$ in (7) if it satisfies the Euler Lagrange equation

$$
L_{1}^{\sigma}(t)+\left({ }_{b} \nabla^{\alpha} L_{2}\right)(t)=0, \quad t \in \mathbb{N}_{a+1, b-2},
$$

where $L_{1}=\frac{\partial g}{\partial f^{\rho}}$ and $L_{2}=\frac{\partial g}{\partial^{C} \nabla_{a}^{\alpha} f}$.

Theorem 3. Let $f$ be a local minimum for $J$ in (5), subject to the boundary conditions (6) and the isoperimetric constraint (7). Assume that $f$ is not an extremal for I. Then there exists a constant $\lambda$ such that $f$ satisfies

$$
\widetilde{L}_{1}^{\sigma}(t)+\left({ }_{b} \nabla^{\alpha} \widetilde{L}_{2}\right)(t)=0, \quad t \in \mathbb{N}_{a+1, b-2},
$$

where $F=L-\lambda g, \widetilde{L}_{1}=\frac{\partial F}{\partial f^{\rho}}$ and $\widetilde{L}_{2}=\frac{\partial F}{\partial^{C} \nabla_{a}^{\alpha} f}$.

Proof. (See also [18]). Let $\eta_{k}: \mathbb{N}_{a, b-1} \rightarrow \mathbb{R}, \eta_{k}(a)=\eta_{k}(b-1)=0$ be two functions and $\epsilon_{k} \mathrm{~s}$ be two arbitrarily small real numbers for $k=1,2$. Consider the new function of two parameters

$$
\hat{f}=f+\epsilon_{1} \eta_{1}+\epsilon_{2} \eta_{2} .
$$

Let

$$
\begin{aligned}
\hat{I}\left(\epsilon_{1}, \epsilon_{2}\right)= & I(\hat{f})-l \\
= & \sum_{t=a+1}^{b-1} g\left(t, f^{\rho}(t)+\epsilon_{1} \eta_{1}^{\rho}(t)+\epsilon_{2} \eta_{2}^{\rho}(t),\left({ }^{C} \nabla_{a}^{\alpha} f\right)(t)+\epsilon_{1}\left({ }^{C} \nabla_{a}^{\alpha} \eta_{1}\right)(t)\right. \\
& \left.+\epsilon_{2}\left({ }^{C} \nabla_{a}^{\alpha} \eta_{2}\right)(t)\right)-l .
\end{aligned}
$$

We have

$$
\begin{aligned}
& \left.\frac{\partial \hat{I}}{\partial \epsilon_{2}}\right|_{(0,0)}= \\
& \sum_{t=a+1}^{b-1}\left[\eta_{2}^{\rho}(t) \frac{\partial g}{\partial f^{\rho}}\left(t, f^{\rho}(t),\left({ }^{C} \nabla_{a}^{\alpha} f\right)(t)\right)+\left({ }^{C} \nabla_{a}^{\alpha} \eta_{2}\right)(t) \frac{\partial g}{\partial^{C} \nabla_{a}^{\alpha} f}\left(t, f f^{\rho}(t),\left({ }^{C} \nabla_{a}^{\alpha} f\right)(t)\right)\right] .
\end{aligned}
$$


Now, by the integration by parts formula (1), we see that

$$
\begin{aligned}
\left.\frac{\partial \hat{I}}{\partial \epsilon_{2}}\right|_{(0,0)}= & \sum_{t=a+1}^{b-1} \eta_{2}^{\rho}(t)\left[\frac{\partial g}{\partial f^{\rho}}\left(t, f^{\rho}(t),\left({ }^{C} \nabla_{a}^{\alpha} f\right)(t)\right)\right. \\
& \left.+\left({ }_{b} \nabla^{\alpha} \frac{\partial g}{\partial^{C} \nabla_{a}^{\alpha} f}\left(t, f^{\rho}(t),\left({ }^{C} \nabla_{a}^{\alpha} f\right)(t)\right)\right)(t-1)\right] \\
& +\left.\eta_{2}(t){ }_{b} \nabla^{-(1-\alpha)}\left(\frac{\partial g}{\partial^{C} \nabla_{a}^{\alpha} f}\left(t, f^{\rho}(t),\left({ }^{C} \nabla_{a}^{\alpha} f\right)(t)\right)\right)(t)\right|_{a} ^{b-1} \\
= & \sum_{t=a+1}^{b-1} \eta_{2}^{\rho}(t)\left[\frac{\partial g}{\partial f \rho}\left(t, f^{\rho}(t),\left({ }^{C} \nabla_{a}^{\alpha} f\right)(t)\right)\right. \\
& \left.+\left({ }_{b} \nabla^{\alpha} \frac{\partial g}{\partial^{C} \nabla_{a}^{\alpha} f}\left(t, f^{\rho}(t),\left({ }^{C} \nabla_{a}^{\alpha} f\right)(t)\right)\right)(t-1)\right] .
\end{aligned}
$$

Since $f$ is not an extremal for $I$, there exists a function $\eta_{2}$ such that

$$
\left.\frac{\partial \hat{I}}{\partial \epsilon_{2}}\right|_{(0,0)} \neq 0 \text {. }
$$

From (9) and the fact that $\hat{I}(0,0)=0$, by the Implicit Function Theorem, it follows that there exists a $C^{1}$ function $\epsilon_{2}=\epsilon_{2}\left(\epsilon_{1}\right)$ defined in a neighborhood of zero such that $\hat{I}\left(\epsilon_{1}, \epsilon_{2}\left(\epsilon_{1}\right)\right)=0$. Therefore, there exists a family of variations of type $(8)$ that satisfy the isoperimetric constraint (7).

Similarly, lets now define

$$
\hat{J}\left(\epsilon_{1}, \epsilon_{2}\right)=J(\hat{f}) .
$$

Since $\hat{J}$ has an extremum at $(0,0)$ subject to the constraint $\hat{I}(0,0)=0$ and since $\nabla \hat{I}(0,0) \neq \mathbf{0}$, by the Lagrange Multiplier Rule, there is a number $\lambda$ such that

$$
\nabla(\hat{J}(0,0)-\lambda \hat{I}(0,0))=\mathbf{0} .
$$

It can easily be shown that

$$
\begin{aligned}
\left.\frac{\partial \hat{J}}{\partial \epsilon_{1}}\right|_{(0,0)}= & \sum_{t=a+1}^{b-1} \eta_{1}^{\rho}(t)\left[\frac{\partial L}{\partial f^{\rho}}\left(t, f^{\rho}(t),\left({ }^{C} \nabla_{a}^{\alpha} f\right)(t)\right)\right. \\
& \left.+\left({ }_{b} \nabla^{\alpha} \frac{\partial L}{\partial^{C} \nabla_{a}^{\alpha} f}\left(t, f^{\rho}(t),\left({ }^{C} \nabla_{a}^{\alpha} f\right)(t)\right)\right)(t-1)\right]
\end{aligned}
$$

and

$$
\begin{aligned}
\left.\frac{\partial \hat{I}}{\partial \epsilon_{1}}\right|_{(0,0)}= & \sum_{t=a+1}^{b-1} \eta_{1}^{\rho}(t)\left[\frac{\partial g}{\partial f^{\rho}}\left(t, f^{\rho}(t),\left({ }^{C} \nabla_{a}^{\alpha} f\right)(t)\right)\right. \\
& \left.+\left({ }_{b} \nabla^{\alpha} \frac{\partial g}{\partial^{C} \nabla_{a}^{\alpha} f}\left(t, f^{\rho}(t),\left({ }^{C} \nabla_{a}^{\alpha} f\right)(t)\right)\right)(t-1)\right] .
\end{aligned}
$$


Hence, we have

$$
\sum_{t=a+1}^{b-1} \eta_{1}^{\rho}(t)\left[\frac{\partial F}{\partial f^{\rho}}\left(t, f^{\rho}(t),\left({ }^{C} \nabla_{a}^{\alpha} f\right)(t)\right)+\left({ }_{b} \nabla^{\alpha} \frac{\partial F}{\partial^{C} \nabla_{a}^{\alpha} f}\left(t, f^{\rho}(t),\left({ }^{C} \nabla_{a}^{\alpha} f\right)(t)\right)\right)(t-1)\right] .
$$

Since $\eta_{1}$ is arbitrary, we obtain

$$
\begin{array}{r}
\frac{\partial F}{\partial f^{\rho}}\left(t, f^{\rho}(t),\left({ }^{C} \nabla_{a}^{\alpha} f\right)(t)\right)+\left({ }_{b} \nabla^{\alpha} \frac{\partial F}{\partial^{C} \nabla_{a}^{\alpha} f}\left(t, f^{\rho}(t),\left({ }^{C} \nabla_{a}^{\alpha} f\right)(t)\right)\right) \\
(t-1)=0 \\
t \in \mathbb{N}_{a+2, b-1}
\end{array}
$$

Now, lets consider the particular case, where the functionals $\widetilde{J}$ and $\widetilde{I}$ are defined by

$$
\widetilde{J}(f)=\sum_{t=a+1}^{b-1}\left[p(t)\left(\left({ }^{C} \nabla_{a}^{\alpha} f\right)(t)\right)^{2}+q(t-1) f^{2}(t-1)\right]
$$

and

$$
\widetilde{I}(f)=\sum_{t=a}^{b-2} r(t) f^{2}(t)=1
$$

respectively. Now proceeding as in the proofs of Theorem 2.5 and Theorem 2.7 in [17], we obtain the following results.

Theorem 4. Let $\lambda_{1}$ be the first eigenvalue of the SLP (2)-(3), and let $x_{1}$ be the corresponding eigenfunction normalized to satisfy the isoperimetric constraint (11). Then $x_{1}$ is the minimum of the functional $\widetilde{J}$ defined by equation (10) subject to the boundary conditions (3) and the isoperimetric constraint (11). Furthermore, $\widetilde{J}\left(x_{1}\right)=$ $\lambda_{1}$.

Proof. Assume that $x$ is a local minimum of $\widetilde{J}$. First note that the Euler Lagrange equation for $\widetilde{I}$ is

$$
2 r(t) x(t)=0, \quad t \in \mathbb{N}_{a+1, b-2},
$$

which is satisfied only for the trivial solution $x(t)=0$ since $r(t)>0$. Hence, no extremals for $\widetilde{I}$ can satisfy the isoperimetric constraint (11). Then, by Theorem 3 , there exists a real constant $\lambda$ such that $x$ satisfies the equation

$$
\left({ }_{b} \nabla^{\alpha}\left(p^{C} \nabla_{a}^{\alpha} x\right)\right)(t)+q(t) x(t)-\lambda r(t) x(t)=0, \quad t \in \mathbb{N}_{a+1, b-2},
$$

together with $x(a)=x(b-1)=0$ and the isoperimetric constraint (11). Let us multiply (12) by $x(t)$ and sum from $a+1$ to $b-2$, then

$$
\sum_{t=a+1}^{b-2} x(t)\left({ }_{b} \nabla^{\alpha}\left(p^{C} \nabla_{a}^{\alpha} x\right)\right)(t)+\sum_{t=a+1}^{b-2} q(t) x^{2}(t)=\lambda \sum_{t=a+1}^{b-2} r(t) x^{2}(t) .
$$


Using $x(a)=x(b-1)=0$, by the integration by parts formula (1), we have

$$
\begin{aligned}
\sum_{t=a+1}^{b-2} x(t)\left({ }_{b} \nabla^{\alpha}\left(p^{C} \nabla_{a}^{\alpha} x\right)\right)(t)= & -\left.x(t)\left({ }_{b} \nabla^{-(1-\alpha)}\left(p^{C} \nabla_{a}^{\alpha} x\right)\right)(t)\right|_{a} ^{b-1} \\
& +\sum_{t=a+1}^{b-1} p(t)\left(\left({ }^{C} \nabla_{a}^{\alpha} x\right)(t)\right)^{2} \\
= & \sum_{t=a+1}^{b-1} p(t)\left(\left({ }^{C} \nabla_{a}^{\alpha} x\right)(t)\right)^{2} .
\end{aligned}
$$

From (11) and the fact that $x(a)=0$, we see that $\widetilde{J}(x)=\lambda$. Since $x$ satisfies $(11)$, it follows that $x$ is nontrivial. Hence, $\lambda$ is an eigenvalue. From Theorem 2, we know that there exists the least element in the spectrum, the first eigenvalue $\lambda_{1}$, and a corresponding eigenfunction $x_{1}$ normalized to satisfy the isoperimetric constraint (11). Hence, the minimum value of $\widetilde{J}$ is $\lambda_{1}$ and $\widetilde{J}\left(x_{1}\right)=\lambda_{1}$.

Definition 3. We will call the functional $R$ defined by

$$
R(x)=\frac{\widetilde{J}(x)}{\widetilde{I}(x)},
$$

where $\widetilde{J}$ and $\widetilde{I}$ are the functionals defined by (10) and (11), respectively, as the Rayleigh quotient for the $S L P(2)-(3)$.

Theorem 5. Assume that $x$ satisfies the boundary conditions $x(a)=x(b-1)=0$ and nontrivial.

(i) If $x$ is a minimizer of the Rayleigh quotient $R$ for the SLP (2)-(3), then the minimum value of $R$ is the smallest eigenvalue $\lambda_{1}$; that is, $R(x)=\lambda_{1}$.

(ii) If $x$ is a maximizer of the Rayleigh quotient $R$ for the SLP (2)-(3), then the maximum value of $R$ is the largest eigenvalue $\lambda_{N-1}$; that is, $R(x)=\lambda_{N-1}$.

Proof. We will only prove (i). The proof of (ii) is similar. Assume that $x$ satisfies the boundary conditions $x(a)=x(b-1)=0$, nontrivial, and is a minimizer of the Rayleigh quotient $R$. We define the functions $\phi, \psi$ and $\zeta$ as follows:

$$
\begin{aligned}
\phi:(-\epsilon, \epsilon) \rightarrow \mathbb{R}, \quad \phi(h)=\widetilde{I}(x+h \eta)= & \sum_{t=a}^{b-2} r(t)(x+h \eta)^{2}(t) \\
\psi:(-\epsilon, \epsilon) \rightarrow \mathbb{R}, \quad \psi(h)=\widetilde{J}(x+h \eta)= & \sum_{t=a+1}^{b-1}\left[p(t)\left(\left({ }^{C} \nabla_{a}^{\alpha}(x+h \eta)\right)(t)\right)^{2}\right. \\
& \left.+q(t-1)(x+h \eta)^{2}(t-1)\right] \\
& \widetilde{J}(x+h \eta) \\
\zeta:(-\epsilon, \epsilon) \rightarrow \mathbb{R}, \quad \zeta(h)=R(x+h \eta)= & \frac{\widetilde{I}(x+h \eta)}{\widetilde{I}(x)}
\end{aligned}
$$


where $\eta: \mathbb{N}_{a, b-1} \rightarrow \mathbb{R}, \eta(a)=\eta(b-1)=0$. Note that ${ }^{C} \nabla_{a}^{\alpha}$ is a linear operator, hence, we have

$$
\left({ }^{C} \nabla_{a}^{\alpha}(x+h \eta)\right)(t)=\left({ }^{C} \nabla_{a}^{\alpha} x\right)(t)+h\left({ }^{C} \nabla_{a}^{\alpha} \eta\right)(t) .
$$

Since $\zeta \in C^{1}((-\epsilon, \epsilon))$ and

$$
\zeta(0) \leq \zeta(h),|h|<\epsilon,
$$

it follows that

$$
\zeta^{\prime}(0)=\left.\frac{d}{d h} R(x+h \eta)\right|_{h=0}=0 .
$$

Moreover, we have

$$
\begin{aligned}
\zeta^{\prime}(h) & =\frac{1}{\phi(h)}\left(\psi^{\prime}(h)-\frac{\psi(h)}{\phi(h)} \phi^{\prime}(h)\right), \\
\psi^{\prime}(0) & =\left.\frac{d}{d h} \widetilde{J}(x+h \eta)\right|_{h=0} \\
& =2 \sum_{t=a+1}^{b-1}\left[p(t)\left({ }^{C} \nabla_{a}^{\alpha} x\right)(t)\left({ }^{C} \nabla_{a}^{\alpha} \eta\right)(t)+q(t-1) x(t-1) \eta(t-1)\right], \\
\phi^{\prime}(0) & =\left.\frac{d}{d h} \widetilde{I}(x+h \eta)\right|_{h=0}=2 \sum_{t=a}^{b-2} r(t) x(t) \eta(t) .
\end{aligned}
$$

Hence,

$$
\begin{aligned}
\zeta^{\prime}(0)= & \frac{2}{\widetilde{I}(x)}\left[\sum_{t=a+1}^{b-1}\left[p(t)\left({ }^{C} \nabla_{a}^{\alpha} x\right)(t)\left({ }^{C} \nabla_{a}^{\alpha} \eta\right)(t)+q(t-1) x(t-1) \eta(t-1)\right]\right. \\
& \left.-\frac{\widetilde{J}[x]}{\widetilde{I}[x]} \sum_{t=a}^{b-2} r(t) x(t) \eta(t)\right] \\
= & 0 .
\end{aligned}
$$

Using $\eta(a)=\eta(b-1)=0$, by the integration by parts formula (1), we get

$$
\begin{aligned}
& \sum_{t=a+1}^{b-1} p(t)\left({ }^{C} \nabla_{a}^{\alpha} x\right)(t)\left({ }^{C} \nabla_{a}^{\alpha} \eta\right)(t) \\
= & \left.\eta(t)\left({ }_{b} \nabla^{-(1-\alpha)}\left(p^{C} \nabla_{a}^{\alpha} x\right)\right)(t)\right|_{a} ^{b-1}+\sum_{t=a+1}^{b-1} \eta(t-1)\left({ }_{b} \nabla^{\alpha}\left(p^{C} \nabla_{a}^{\alpha} x\right)\right)(t-1) \\
= & \sum_{t=a+1}^{b-1} \eta(t-1){ }_{b} \nabla^{\alpha}\left(p^{C} \nabla_{a}^{\alpha} x\right)(t-1) .
\end{aligned}
$$

Keeping in mind that $\frac{\widetilde{J}[x]}{\widetilde{I}[x]}=\lambda$ and $\eta(a)=\eta(b-1)=0$, we obtain

$$
\sum_{t=a+1}^{b-2}\left[\left({ }_{b} \nabla^{\alpha}\left(p^{C} \nabla_{a}^{\alpha} x\right)\right)(t)+q(t) x(t)-\lambda r(t) x(t)\right] \eta(t)=0 .
$$


Since $\eta$ is arbitrary, we get

$$
\left({ }_{b} \nabla^{\alpha}\left(p^{C} \nabla_{a}^{\alpha} x\right)\right)(t)+q(t) x(t)-\lambda r(t) x(t)=0, \quad t \in \mathbb{N}_{a+1, b-2} .
$$

Since $x$ is nontrivial, it follows that $\lambda$ is an eigenvalue of (13). In addition, if $\lambda_{i}$ is an eigenvalue and $x_{i}$ is the corresponding eigenfunction, then

$$
\left({ }_{b} \nabla^{\alpha}\left(p^{C} \nabla_{a}^{\alpha} x_{i}\right)\right)(t)+q(t) x_{i}(t)=\lambda_{i} r(t) x_{i}(t), \quad t \in \mathbb{N}_{a+1, b-2} .
$$

As can be seen from the proof of Theorem 4, we have

$$
\lambda_{i}=\frac{\sum_{t=a+1}^{b-1}\left[p(t)\left(\left({ }^{C} \nabla_{a}^{\alpha} x_{i}\right)(t)\right)^{2}+q(t-1) x_{i}^{2}(t-1)\right]}{\sum_{t=a}^{b-2} r(t) x_{i}^{2}(t)} ;
$$

that is, $R\left[x_{i}\right]=\frac{\widetilde{J}\left[x_{i}\right]}{\widetilde{I}\left[x_{i}\right]}=\lambda_{i}$. Since $\lambda$ is the minimum value of $R$, it follows that

$$
\lambda \leq \lambda_{i}, \quad 1 \leq i \leq N-1
$$

Hence, $\lambda=\lambda_{1}$.

\section{ACKNOWLEDGEMENTS}

This study was supported by The Scientific and Technological Research Council of Turkey while the first author visiting the University of Nebraska-Lincoln. The third author would like to thank Prince Sultan University for funding this work through research group Nonlinear Analysis Methods in Applied Mathematics (NAMAM) group number RG-DES-2017-01-17.

\section{REFERENCES}

[1] I. Podlubny, Fractional Differential Equations, Academic Press, San Diego CA, 1999.

[2] G. Samko, A. A. Kilbas and S. Marichev, Fractional Integrals and Derivatives, Theory and Applications, Gordon and Breach, Yverdon, 1993.

[3] A. A. Kilbas, M. H. Srivastava and J. J. Trujillo, Theory and Application of Fractional Differential Equations, North Holland Mathematics Studies 204, 2006.

[4] R. L. Magin, Fractional Calculus in Bioengineering, Begell House Publishers, 2006.

[5] D. Baleanu, K. Diethelm, E. Scalas and J. J. Trujillo, Fractional Calculus Models and Numerical Methods, (Series on Complexity, Nonlinearity and Chaos), World Scientific, 2012. 
[6] C. Goodrich, Allan C. Peterson, Discrete fractional calculus, Springer (2015).

[7] T. Abdeljawad, On Riemann and Caputo fractional differences, Comput. Math. Appl. 62, 1602-1611 (2011).

[8] F. M. Atıcı and P. W. Eloe, A Transform method in discrete fractional calculus, Int. J. Differ. Equ. 2, 165-176 (2007).

[9] F. M. Atıcı and P. W. Eloe, Discrete fractional calculus with the nabla operator, Electr. J. Qualit. Theor. Differ. Equ. 1-12 (2009).

[10] H. L. Gray and N. F.Zhang, On a new definition of the fractional difference, Math. Comput. 50, 513-529 (1988).

[11] T. Abdeljawad and F. Atici, On the definitions of nabla fractional differences, Abstr. Appl. Anal. Volume 2012 (2012), Article ID 406757, 13 pages doi, $10.1155 / 2012 / 406757$.

[12] T. Abdeljawad, Dual identities in fractional difference calculus within Riemann, Adv. Differ. Equ. 2013, 2013, 36.

[13] T. Abdeljawad, On Delta and Nabla Caputo fractional differences and dual identities, Discr. Dyn. Nat. Soc. Volume 2013 (2013), Article ID 406910, 12 pages.

[14] B.V. Brunt, The Calculus of Variations, Springer (2003).

[15] T. Abdeljawad, Nabla Euler-Lagrange equations in discrete fractional variational calculus within Riemann and Caputo, Int. J. Math. Comput. 22, 144-153 (2014).

[16] T. Abdeljawad and D. Baleanu, On Fractional Derivatives with Exponential Kernel and Their Discrete Versions, Reports on Mathematical Physics, 80, 11-27 (2017).

[17] R. Almeida, A.B. Malinowska, M.L. Morgado and T. Odzijewicz, Variational methods for the solution of fractional discrete/continuous Sturm-Liouville problems, J. Mech. Mater. Struct., Doi, 10.2140/jomms.2017.12.3.

[18] R. Almeida, D.F.M. Torres, Necessary and Sufficient Conditions for the Fractional Calculus of Variations with Caputo Derivatives, Commun. Nonlinear Sci. Numer. Simul. 16(3), 1490-1500 (2011).

[19] N.R.O. Bastos, R.A.C. Ferreira and D.F.M. Torres, Discrete-time fractional variational problems, Signal Proc. 91, 513-524 (2011).

[20] O. P. Agrawal, Formulation of Euler-Lagrange equations for fractional variational problems, J. Math. Anal. Appl. 272,(2002) 368-374.

[21] O. P. Agrawal, Generalized Euler-Lagrange Equations and Transversality Conditions for FVPs in terms of Caputo Derivative, J. Vib. Control 13(9-10),(2007) 1269-1281. 
[22] D. Baleanu, T. Abdeljawad, F. Jarad, Fractional variational principles with delay, Journal of Physica A, Math. and Theor., 41(31), (2008), 315-403.

[23] F. Jarad, D. Baleanu, T. Abdeljawad, Fractional variational principles with delay within Caputo derivatives, Reports on Mathematical Physics, 65, (2010), 17-28.

[24] F. Jarad, D. Baleanu, T. Abdeljawad, Fractional variational optimal control problems with delayed arguments, Nonlinear Dynamics, 62, (2010), 609-614.

[25] F. Jarad, T. Abdeljawad, D. Baleanu, Higher order fractional variational optimal control problems with delayed arguments, Applied Mathematics and Computation, 218(18), (2012), 9234-9240.

[26] F. Jarad, T. Abdeljawad, D. Baleanu, On Riesz-Caputo formulation for sequential fractional variational principles, Abstract and Applied Analysis, (2012), Volume 2012, Article ID 890396, 15 pages.

[27] T. Abdeljawad, D. Baleanu, Fractional differences and integration by parts, J. Comput. Anal. Appl. 13(3), (2011) 574-582.

[28] T. Abdeljawad, D. Baleanu, Integration by parts and its application of a new nonlocal fractional derivative with Mittag-Leffler kernel, J. Nonlinear Sci. Appl., 10, (2017), 1098-1107.

[29] D. Baleanu, J. J. Trujillo, On exact solutions of a class of fractional EulerLagrange equations, Nonlin.Dyn. 52(4), (2008) 331-335.

[30] R. Almeida, Variational problems involving a Caputo-type fractional derivative, J. of Optim. Theory Appl., 147, (2017), 276-294.

[31] Matheus J.Lazo, Delfim F.M. Torres, Variational calculus with confromable fractional derivatives, to appear in IEEE/CAA Journal of Automatica Sinicia.

[32] W. E. Boyce, R.C. DiPrima, Elementary differential equations and boundary value problems (John Wiley and Sons, USA, 2005).

[33] A. Zettl, Sturm Liouville Theory, in Mathematical Surveys and Monographs, vol. 121, American Mathematical Society, 2005.

[34] M. Al-Refai, T. Abdeljawad, Fundamental Results of Comformable Sturm-Liouville Eigenvalue Problems, Complexity 2017 (2017), Article ID 3720471, 7 pages.

[35] M. Klimek, O.P. Agrawal, Fractional Sturm Liouville problem, Computers and Mathematics with Applications 66 (2013) 795-812.

[36] M. Rivero, J.J. Trujillo, M. P. Velasco, A fractional approach to the SturmLiouville problem, Cent. eur. J. Phys. 11 (10) 2013, 1246-1254. 